in the hospice for a short period of time it is vital that we know what matters to them and what is important to them not just their clinical condition. We are encouraging staff to discuss one page profiles with patients on admission so that staff can get to know their wishes right from the start. Patients can change their one page profiles at any time.

We are receiving very positive feedback from patients that have completed them. Comments include:

'I feel much more relaxed now that you know what my likes and dislikes are'

'Suddenly after completing the one page profile I feel I have the strength to talk to my daughter about my wishes'

'I am happy that my wife will be involved with decisions about my care this means a lot to me'

'I feel safe and secure that my wish not to be resuscitated has been listened to and everyone will know my wishes'

We have a working group who are undertaking evaluations and will audit this work.

\section{P-124 PREFERRED PLACE OF CARE - ROOT CAUSE ANALYSIS}

${ }^{1}$ Maria Debattista, ${ }^{1}$ Andrew Fletcher, ${ }^{2}$ Claire Capewell. ${ }^{1}$ St Catherine's Hospice, Preston, UKi ${ }^{2}$ Lancashire Teaching Hospitals Trust

\subsection{6/bmjspcare-2016-001245.147}

Introduction Surveys and research indicate that home is the preferred place of care at the end of life for many people (71\%), whilst the most commonly recorded place of death remains hospital (53\%).

Aim To identify barriers and enablers to the delivery of good end-of-life care for patients.

Methods The project was completed over a six-week period at a hospice in Northwest England. Each week at our multidisciplinary team meeting we discussed deaths of patients known to our community palliative care team and recorded our findings.

Results 48 patients known to the team died during the six- week period. Preferred place of death was achieved in 91\% (30/33) of cases when this was home, and $85 \%(11 / 13)$ of cases when this was the hospice. None of our patients expressed hospital as their PPD. Preferred place of death was unknown in $4.2 \%(2 / 48)$ of patients. $8.3 \%(4 / 48)$ of patients died in hospital. In 50\% (2/4) patients, a hospital admission was indicated due to acute unexpected deterioration. $77 \%(21 / 27)$ of patients dying in the community had face to face or telephone contact within three days of death. Identified barriers to good end-of-life care were: difficulties accessing oxygen at home for symptom management, one episode of difficulty accessing specialist care advice and challenges communicating directly with district nursing teams. Enablers were: appropriate use of DNACPR forms with no involvement of emergency services at the end-of-life as well as good team-working between specialist and generalist services.

Conclusions The majority of patients known to the team die in their preferred place of care, with a good quality of death as judged by the team. Lessons have been learned from after death analysis and action plans devised. This scoping exercise could be repeated on a periodic basis.
P-125

HOMEWARD BOUND: RAPID DISCHARGE OF PEOPLE TO THEIR PREFERRED PLACE OF CARE

Christina Ginsbu. Earl Mountbatten Hospice, Newport, UK

10.1136/bmjspcare-2016-001245.148

Following a period of sustained pressure on our local healthcare system, the gauntlet was thrown down from commissioners to the hospice to expedite discharges by providing care at home.

Our hospice, at the heart of the community, renowned for breaking barriers and a positive approach, rose to the challenge of this six-month pilot project.

We rapidly reorganised our community team to release their community support workers to form a team and recruited to expand the workforce.

This newly formed team received a week's robust training in preparation for our crusade to get people home from an inpatient setting.

We met with commissioners and local care providers to plan in partnership how we would work.

Aims of the project

- To respond to local need by developing a reactive workforce to deliver flexible, high quality personal and health care to support people at the end of their life

- Enable rapid response discharges by accepting fast track care requests from Continuing Healthcare within 1.5 hours and commencing care within four hours

- Preventing admissions and decreasing the need for use of acute care by providing and sustaining care at home

- Increase the number of people who choose to live and die in their own homes.

Results

- $100 \%$ of referrals were accepted within 1.5 hours

- $100 \%$ of these referrals received care within four hours

- $100 \%$ of these patients died in their preferred place of care

- We have met the needs of $56 \%$ of all fast track care requests.

- $18 \%$ were in partnership with local care providers.

- The evidence from the quality survey completed by families, has demonstrated nothing but positive feedback to the excellent responsiveness of the service delivery.

Conclusion It is the intention of the Hospice to continue and expand this service

\section{P-126 AN AUDIT TO DETERMINE IF PEOPLE WITH A LEARNING DISABILITY (LD) AND KNOWN TO THE HOSPICE HAVE ACHIEVED THEIR PREFERRED PLACE OF DEATH (PPD) AND IF THE INITIAL DISCUSSION HAD TAKEN PLACE, WITH AN OUTCOME ESTABLISHED AND DOCUMENTED}

Maria Stripe, Sue Spong. St Francis Hospice, Romford, UK

\subsection{6/bmjspcare-2016-001245.149}

Aims and objectives To establish documented evidence of the preferred place of death (PPD) of people with a learning disability (LD) and establish if PPD was achieved and documented. Additionally; to establish where initial conversation took place with regards to PPD. To establish if an "easy read" Preferred 
Priorities for Care was used or not. To add some narrative to capture the complexity of caring for each individual with a learning disability.

Background This audit evolved as an outcome of a previous learning disabilities audit in January 2015. Following discussion at a multi-disciplinary team meeting it was agreed that a further audit on an individual's PPD would highlight if the hospice was meeting their objectives within the learning disability pathway under future planning considerations.

Method A qualitative retrospective audit was completed from 12 patient's notes on i-care entries, who were known to have a learning disability over a one year period.

Results All deaths achieved their PPD with 100 percent documented evidence. There was a rich narrative and person-centred care, demonstrating continuity.

Conclusion The results revealed aspiring achievements with significant learning outcomes for the hospice multi-disciplinary teams. Actions identified were: An easy read booklet for preferred priorities for care to be offered where appropriate: To highlight the importance of documenting capacity/PPD discussions where appropriate. Current notes to be reviewed with regards to power of attorney/registration. To promote awareness of the resource folder in the clinical nurse specialist office: To share the importance of the narrative and adherence to the standard in the learning disability pathway.

\section{P-127 ANTIMICROBIAL STEWARDSHIP - IMPROVING PRESCRIBING ON THE INPATIENT UNIT}

Wendy Pepper, Rebecca Bhatia. Dorothy House Hospice Care, Bradford-On-Avon, UK

\subsection{6/bmjspcare-2016-001245.150}

Introduction The Inpatient Unit at Dorothy House Hospice Care committed to comply with national guidelines for antibiotic prescribing. The document followed was the Department of Health's 'Antimicrobial Stewardship: Start Smart Then Focus'. Although the guidance had been produced for hospitals the guiding principles were also applicable in the hospice setting. We also wanted to ensure compliance with Criterion 3 of the Health and Social Care Act 2015 where it states "Ensure appropriate antimicrobial use to optimise patient outcomes and to reduce the risk of adverse events and antimicrobial resistance".

Aims The aims were to ensure Dorothy House was compliant with local and national guidelines on antibiotic prescribing

To identify how antibiotics are prescribed and supplied by Dorothy House and to improve antimicrobial stewardship.

Methods A patient database was set up to capture all patients prescribed antibiotics.

Standards were set to ensure:

- Compliance with local guidelines

- Documented indication for antibiotic use was on the medicine chart

- Documented indication for use was in the patient notes

- The prescription was clear and accurate

- A review date or duration of antibiotic use was prescribed

- Compliance with The Department of Health Advisory Committee on antimicrobial stewardship

- A quarterly audit using the above standards was performed capturing all patients prescribed antibiotics in that time period
Conclusion There was a dramatic improvement in compliance from the quarter one audit to the quarter four audit in all standards but most specifically around documenting an indication for use on prescription charts and setting a review or duration date. There was also a decrease in the use of broad spectrum antibiotics. This audit now forms part of the Dorothy House Hospice Care annual audit plan.

\section{P-128 "EN SUITE DRUGS": QUALITY IMPROVEMENTS IN MEDICINES MANAGEMENT}

Tara Schrikker. St Wilfrid's Hospice, Eastbourne, UK

\subsection{6/bmjspcare-2016-001245.151}

Background The hospice introduced the practice for controlled and non-controlled drugs to be stored and administered from the Patient Own Drug (POD) cupboards located within patients' bedrooms. Further recommendations were addressed after initial implementation to strengthen patient safety.

Aims and objectives

What was the aim of the work?

Increase Productivity and Clinical Effectiveness

For timely medication rounds as staff remain in the patients' rooms at point of administration.

Enhanced Patient Experience

Staff remaining within the patients' rooms provides a visible, reassuring presence.

Quality Improvement and Safety

For stock levels to be tailored to patient need ensuring cost effectiveness

Storing patient's own controlled drugs in PODS reduces the risk of administration incidents.

Method

- Proposal presented to Clinical Governance Committee to introduce PODs in patients' rooms, storing controlled and non-controlled medication.

- Medicines Management Group guided implementation

- Clinical Commissioning Group (CCG) informed of the proposed changes in practice, including the financial implications.

- POD cupboards redesigned and controlled drug cabinets installed to meet legislation and compliance.

- Training sessions held to support staff during change to practice.

- Second phase of implementation included administration of oral controlled drugs from PODs.

Results

- Clinical staff have reported improvement with timely medication rounds.

- Audits found a reduction in the time between the actual prescribed time and patients receiving their oral modified release opioids.

- Patient focused to allow continuation of their preferred administration times.

- Promotion of enabling patients to retain control and independence of their medications.

- Improvement in the management of drug stock levels.

- Decrease in number of related medication incidents in comparison to the previous year.

Conclusion This quality improvement involved all levels of the hospice team. The successful implementation required 